\title{
Role of lung ultrasound in identifying COVID-19 pneumonia in patients with negative swab during the outbreak
}

\author{
Giuseppe Aiosa, Romina Gianfreda, Marco Pastorino, Piero Davio \\ Internal Medicine, Azienda Ospedaliera Santi Antonio e Biagio e Cesare Arrigo, Alessandria, Italy
}

\begin{abstract}
Lung ultrasound is a reasonable tool for detection of manifestations of COVID-19, to facilitate the division of patients flow of infected with SARS-CoV-2 from those affected by other pathologies. Often, a reason for the incorrect separation of the flows is the possibility of false-negative rRT-PCR results.

We aimed to evaluate the advantages of performing Lung Ultrasound (LUS) in patients with a negative swab, to confirm the suspicious of COVID-19 at the bedside, according to the recent findings of typical lung ultrasound lesions of COVID19 related pneumonia. We analyzed 11 non-critical patients admitted to Emergency Department in the Internal Medicine ward, during outbreak, as Covid-19 negative patients affected by pneumonia.

The result of the ultrasound findings conditioned the consequent allocation of the patient. 9/11 patients had typical LUS findings for COVID-19, but only $3 / 11$ patients had a second positive nasopharyngeal swab, and $2 / 11$ had positive swab on pleural fluid. 6/11 patients remained negative with strongly suspicious LUS lesions, and so treated and isolated as Covid-19 positive. 2/11 had negative swab and none LUS findings, thus treated as affected by other pathologies.

These findings clearly show how LUS plays an important role together with the chest $\mathrm{x}$-ray in identifying patients with interstitial pneumonia from COVID-19.
\end{abstract}

\footnotetext{
Correspondence: Piero Davio, Azienda Ospedaliera Santi Antonio e Biagio e Cesare Arrigo, Alessandria, Italy

E-mail: pierodavio@gmail.com

Key words: Lung ultrasound; COVID-19; pneumonia; rRT-PCR; negative swab.

Conflict of interest: No one. This work was not supported by any grant.

Ethics approval and consent to participate: The manuscript does not contain any individual person's data in any form.

Received for publication: 14 April 2020

Accepted for publication: 16 April 2020.

This work is licensed under a Creative Commons Attribution 4.0 License (by-nc 4.0).

(Copyright: the Author(s), 2020

Licensee PAGEPress, Italy

Emergency Care Journal 2020; 16:9026

doi:10.4081/ecj.2020.9026
}

\section{Introduction}

At the beginning of the current COVID-19 pandemic outbreak, many Italian hospitals struggled at dividing the patients flow of infected with SARS-CoV-2 from those affected by other pathologies. Common symptoms at the onset of illness included fever, cough, shortness of breath, pharyngodinia, myalgia, fatigue, headache, and expectoration. ${ }^{1}$

The criteria for identifying the infection in suspicious cases cannot guarantee absolute safety.

If we choose to privilege sensitivity, many uninfected patients will be selected from the Emergency Department (ED) for the area intended for the infected, on the other hand, if we take a particular account of specificity, many infected patients will be sent to the 'clean path'.

A reason for the incorrect separation of the flows was the limited number of rRT-PCR kits and the possibility of false-negative rRT-PCR results since this test sensibility is $71 \%$.

A common mistake in the early stage of the outbreak in our district was to consider uninfected patients those with pneumonia and a negative swab. From the Chinese experience, it appears that the execution of the swab does not detect about $20 \%$ of COVID19 pneumonia. The alternative is the use of chest CT, an essential component in the diagnostic algorithm for patients with suspected COVID-19 infection since this test sensibility is $98 \% .^{2-4}$

Unfortunately, it is often not possible to use CT for patients with suspected COVID 19 pneumonia as the 'clean path' must be preserved. Lung ultrasound has been developed for many years and is now a mature practice, and its application in the diagnosis of severe lung diseases has received considerable recognition. .-9 $^{5}$

A recent publication on the Chinese epidemic outbreak shows the ultrasound image features of non-critical patients with interstitial pneumonia from COVID-19 infection. ${ }^{10}$

\section{Materials and Methods}

\section{Patients}

All patients at the entrance were not critical, had dyspnea and fever, performed serological exams and a chest x-ray with evidence of signs of pneumonia.

We report 11 cases of pneumonia in patients with a first negative rRT-PCR result for COVID-19 infection, admitted to ED in the Internal Medicine ward, and immediately underwent Lung Ultrasound (LUS). The result of the ultrasound findings conditioned the consequent allocation of the patient.

\section{Equipment}

Fujifilm Sonosite Edge II color ultrasonic device was used. The frequency was 2-5 MHz for convex array probe, film and $75 \%$ 
alcohol solution were used to protect and disinfect the equipment. We believe that the use of the higher frequency linear probe 6-13 $\mathrm{MHz}$ can be optional for a higher resolution of the image only in particular cases. The use of a single probe can facilitate sterility maintenance of the devices and reduce the operators' exposition time.

\section{Operational procedures}

LUS exploration of the chest can be done by describing eight sectors $^{9}$ or six sectors on each hemithorax. ${ }^{10}$ We prefer to explore each lung by dividing each hemithorax into six fields to reduce the number of samples and, therefore, the operator's exposure time. Each hemithorax is first divided into three areas, anterior, lateral, and posterior, respectively separated by the anterior and posterior axillary line, then each region is divided into upper and lower. In this manner, the two lungs were divided into 12 areas. (Figure 1).

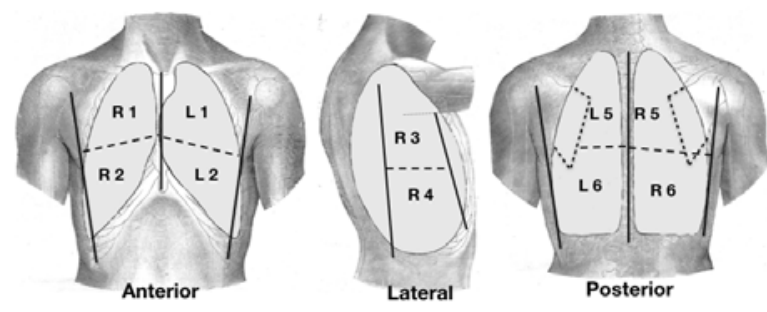

Figure 1. Two lungs divided into 12 areas.

A senior physician with excellent skills and experience in ultrasound performed the LUS assessments. The exposure time to complete the exam is about 3-5 minutes.

The characteristic ultrasound lesions of COVID-19 pneumonia, expected mainly in the posterior lower fields, are sought at the level of the 12 lung areas identified:

i) B lines often fused as white lung under the pleural line (waterfall signs);

ii) thickening and coarseness of the pleural line (main A-line);

iii) subpleural lesions as patchy, strip, and nodule consolidation with air bronchogram;

iv) thickening and edema of parietal pleura with or without localized thin pleural effusion around the lesions.

Other types of lesions as more pleural effusions, empyema, lung hepatization, and lung abscess, are not expected.

\section{Case 1}

An 82-year-old man, C protein reactive (CPR) 8,7 mg/dL (n.v. 0 - 0,8), LDH 572 U/L (n.v. 230-500), Ferritin 464 ng/mL (n.v. 10291) (Figure 2; Figure3A; Figure 3B).

Because the first rRT-PCR result for COVID-19 was negative, the patient was transferred to a protected area. Here, a second swab was positive.

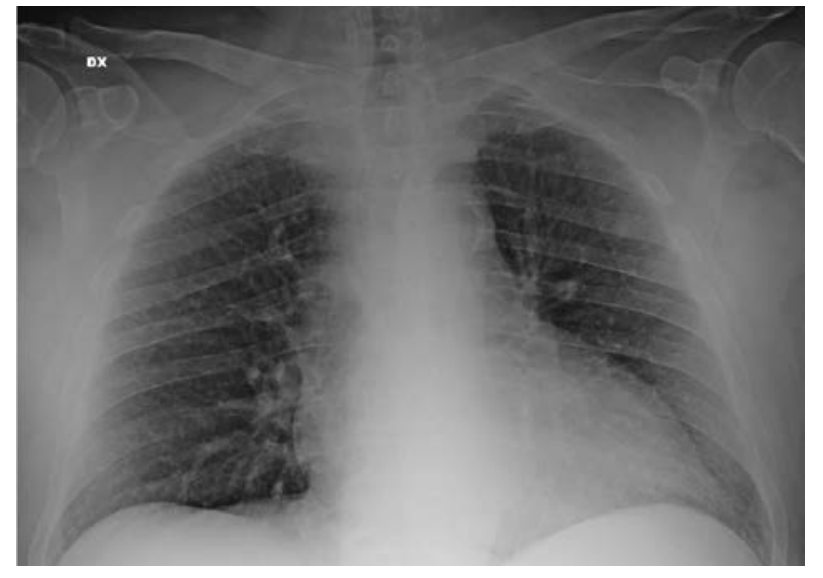

Figure 2. Chest x-ray. Interstitial-alveolar infiltrate with pleural thickening, more evident in the lower-middle fields on the left side.

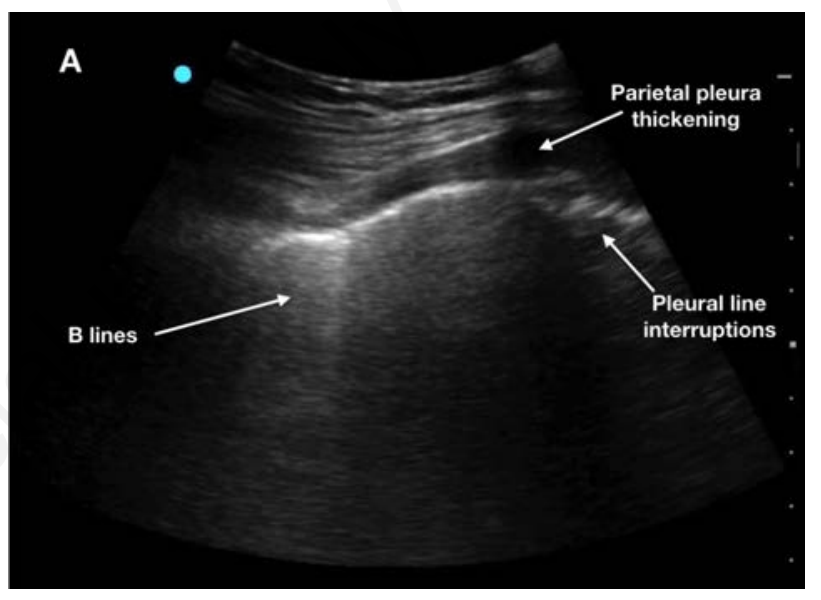

Figure 3A. US imaging. Bilateral thickening of the parietal pleura, thickening, and roughness of the pleural line with interruptions due to the presence of small subpleural infiltrates and $B$ lines. Because the first rRT-PCR result for COVID-19 was negative, the patient was transferred to a protected area. Here, a second swab was positive.

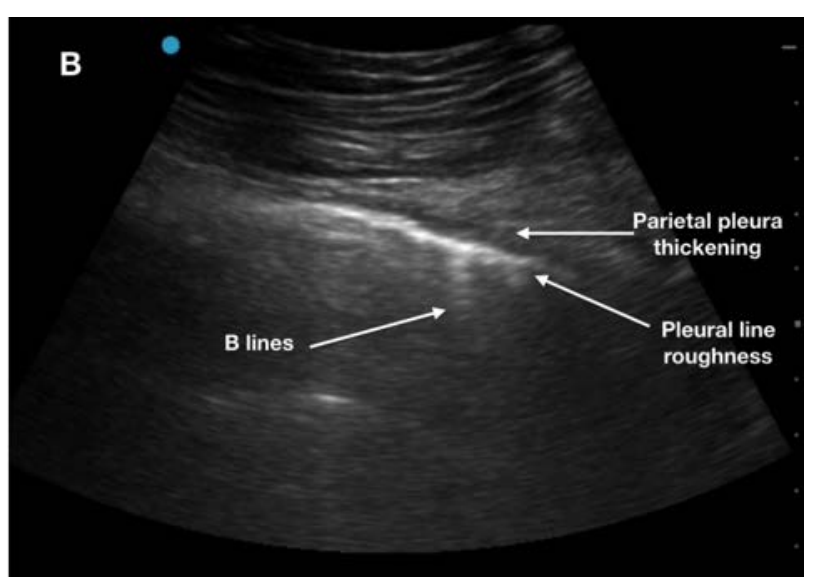

Figure 3B. US imaging. Bilateral thickening of the parietal pleura, thickening, and roughness of the pleural line with interruptions due to the presence of small subpleural infiltrates and $B$ lines. 


\section{Case 2}

A 74-year-old woman, CPR 8,7 mg/dL, LDH 572 U/L, Ferritin 464 ng/mL (Figure 4; Figure 5A; Figure 5B; Figure 5C).

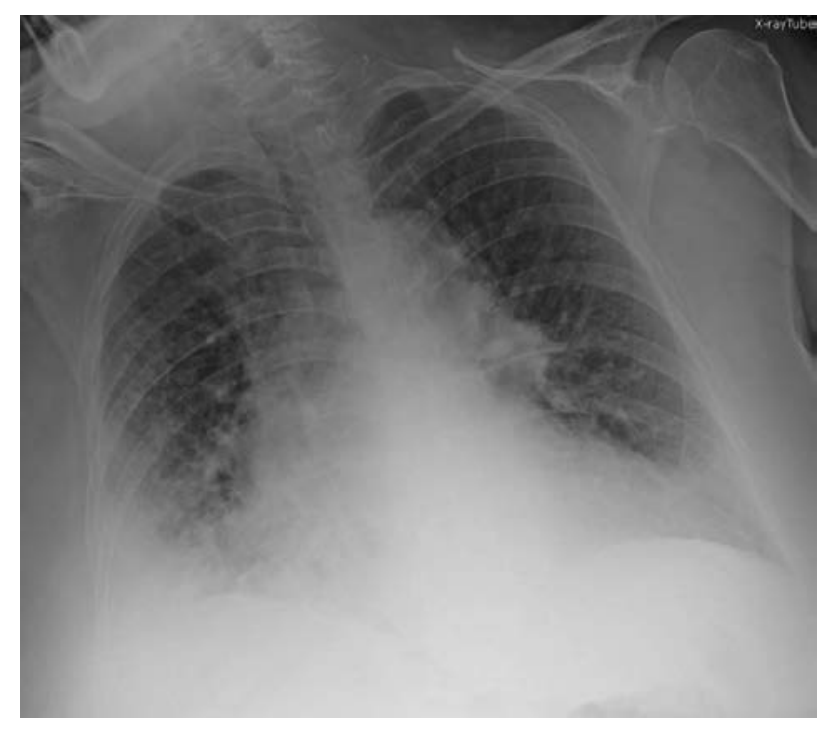

Figure 4. Chest $x$-ray. Consolidation area in the right inferior field with diffuse interstitial texture thickening.

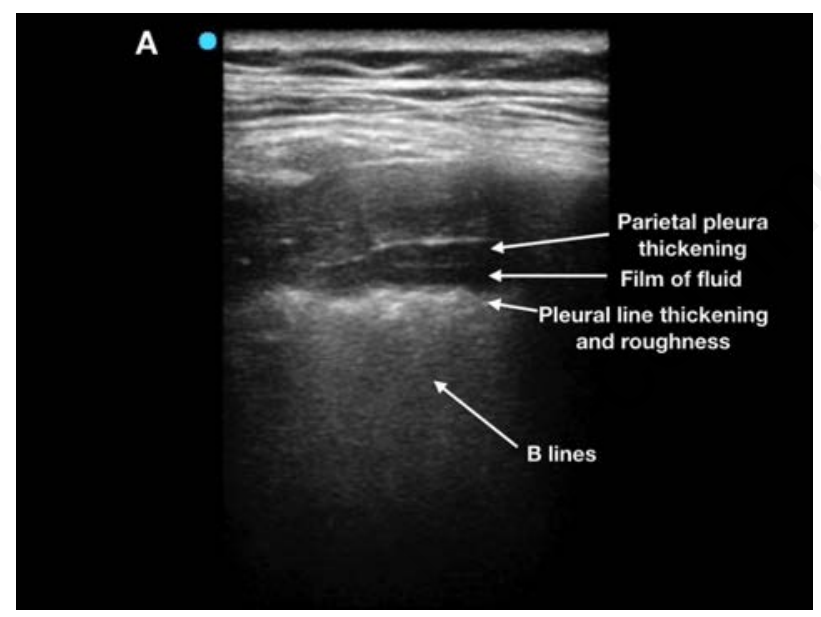

Figure 5A. US imaging. Parietal pleura thickening with a thin film of pleural fluid, thickening, and irregularity of the pleural line with $B$ lines at the right posterior basal field.

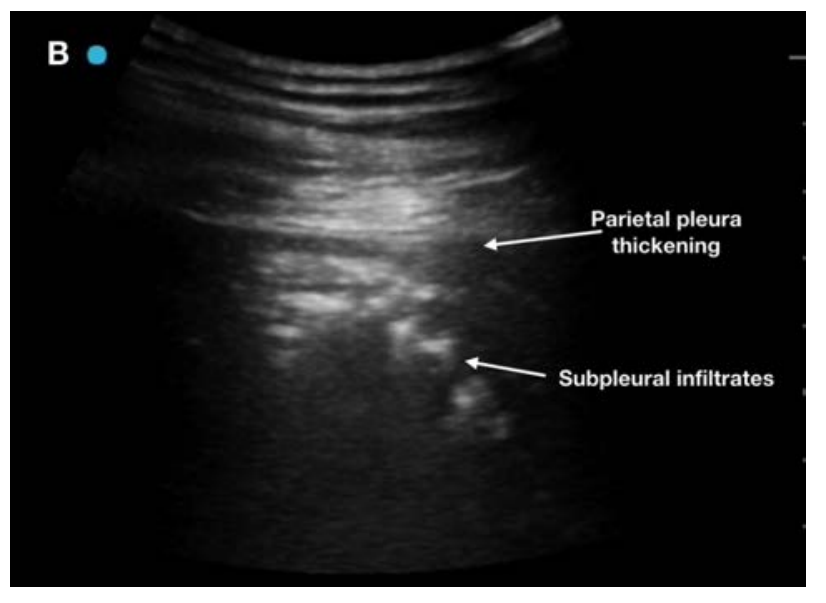

Figure 5B. US imaging. Bilateral subpleural infiltrates.

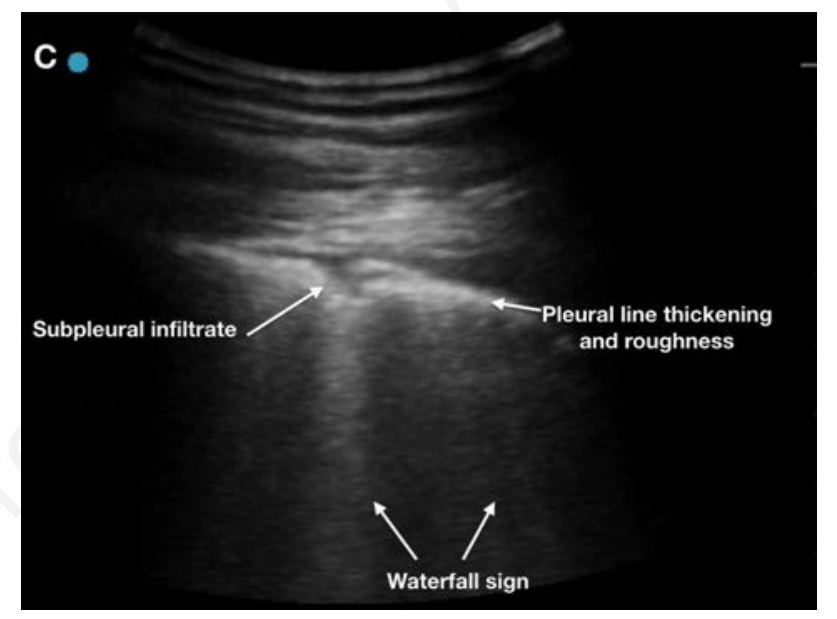

Figure 5C. US imaging. Bilateral posteroinferior thickening and roughness of the pleural line, confluent B lines (waterfall sign) and subpleural infiltrate

Because the second rRT-PCR result for COVID-19 was negative, the patient was transferred to a protected area. 


\section{Case3}

A 75-year-old man, CPR 11,6 mg/dL, LDH 518 U/L, Ferritin 123 ng/mL. (Figure 6; Figure 7A; Figure 7B; Figure 7C).

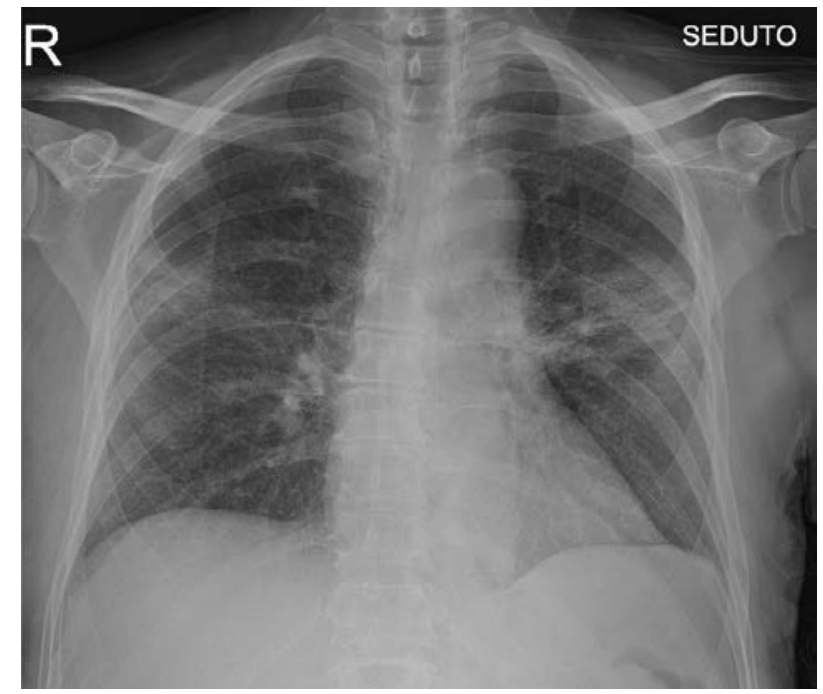

Figure 6. Chest x-ray. Interstitial texture thickening. Nuanced consolidations to the middle fields more evident on the left. Pleural thickening of the upper areas.

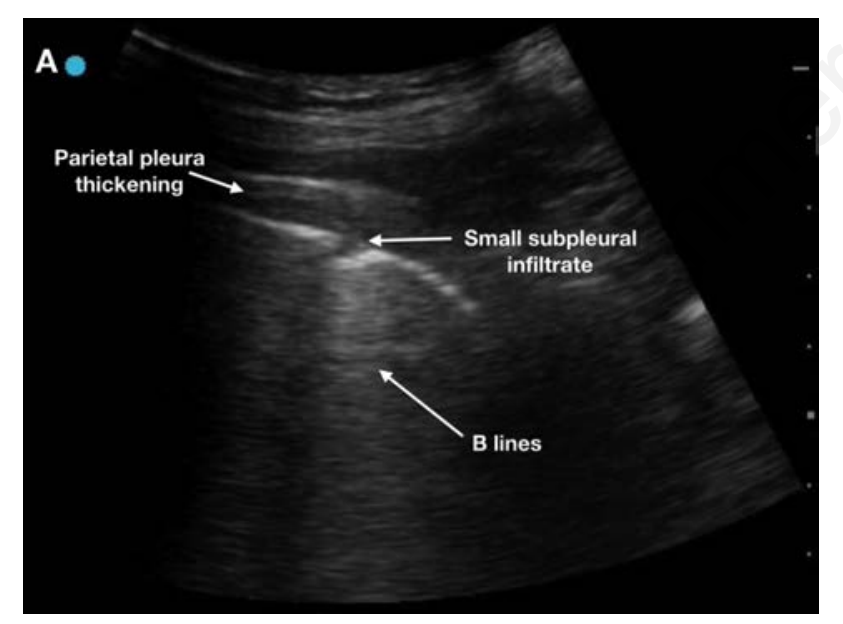

Figure 7A. US imaging. Thickening of the parietal pleura, subpleural infiltrates of various sizes and $B$ lines in bilateral pulmonary posteroinferior fields.

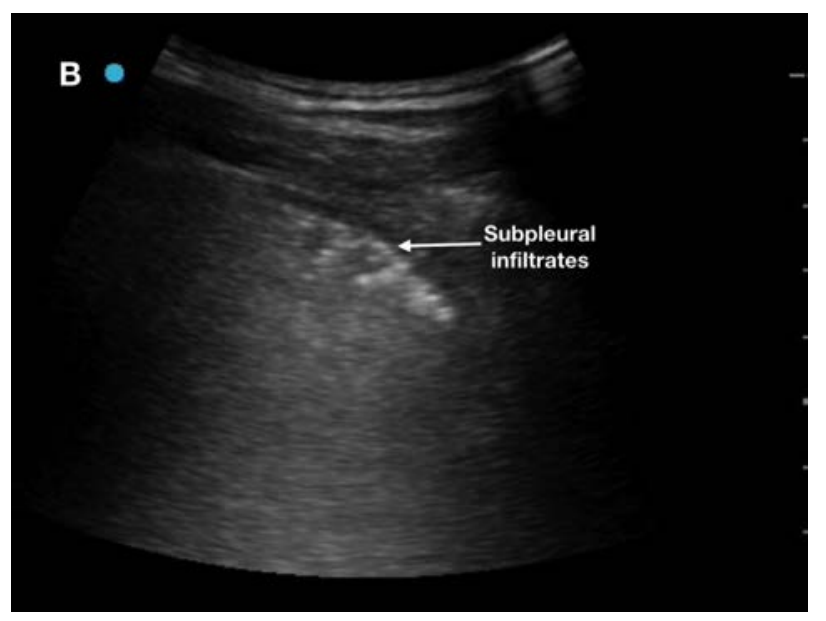

Figure 7B. US imaging. Thickening of the parietal pleura, subpleural infiltrates of various sizes and B lines in bilateral pulmonary posteroinferior fields.

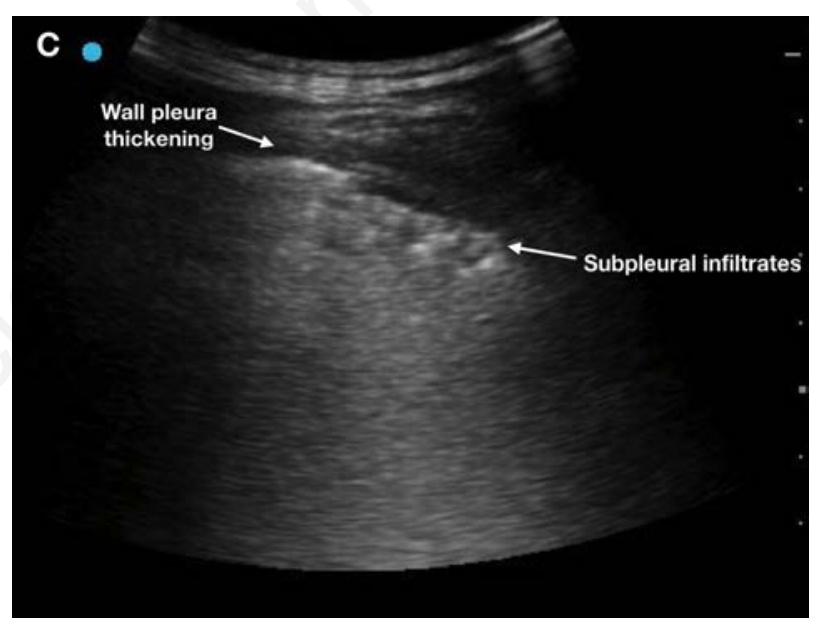

Figure 7C. US imaging. Thickening of the parietal pleura, subpleural infiltrates of various sizes and $B$ lines in bilateral pulmonary posteroinferior fields. 
The US findings were suspected of COVID-19 pulmonary lesions, and then a TC scan was performed (Figure 8).

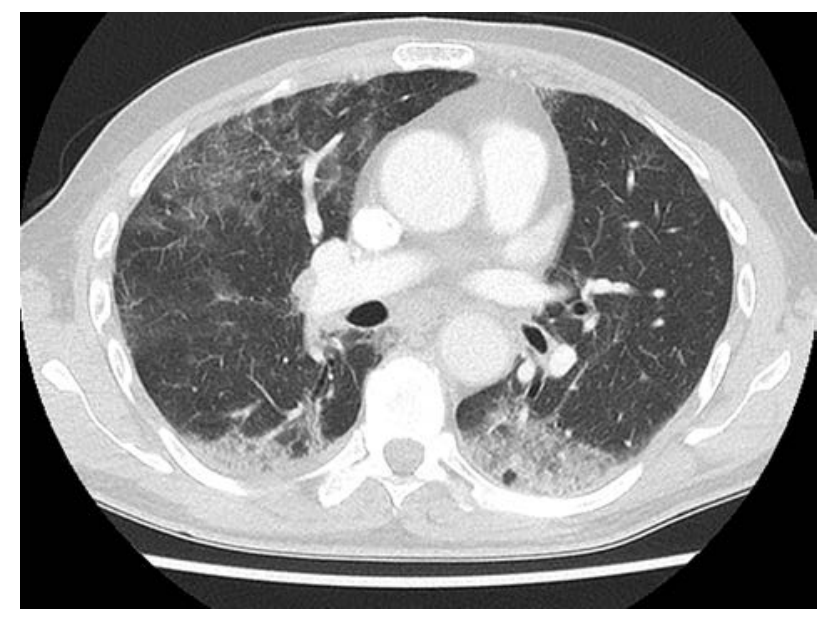

Figure 8. TC imaging. Ground glass areas at the upper fields and consolidation areas at the bilateral posteroinferior fields. Minimal interstitial texture thickening.

A second rRT-PCR result for COVID-19 was negative, and then a Bronchiolar Alveolar Lavage (BAL) was performed with rRT-PCR negative results.

The ultrasound and CT images were suggestive of COVID-19 interstitial-alveolar pneumonia. However, on the base of the negative rRT-PCR results, the patient remained in the ward in a single protected room.

\section{Case 4}

A 92-year-old man, CPR 26 mg/dL (Figure 9; Figure 10A; Figure 10B).

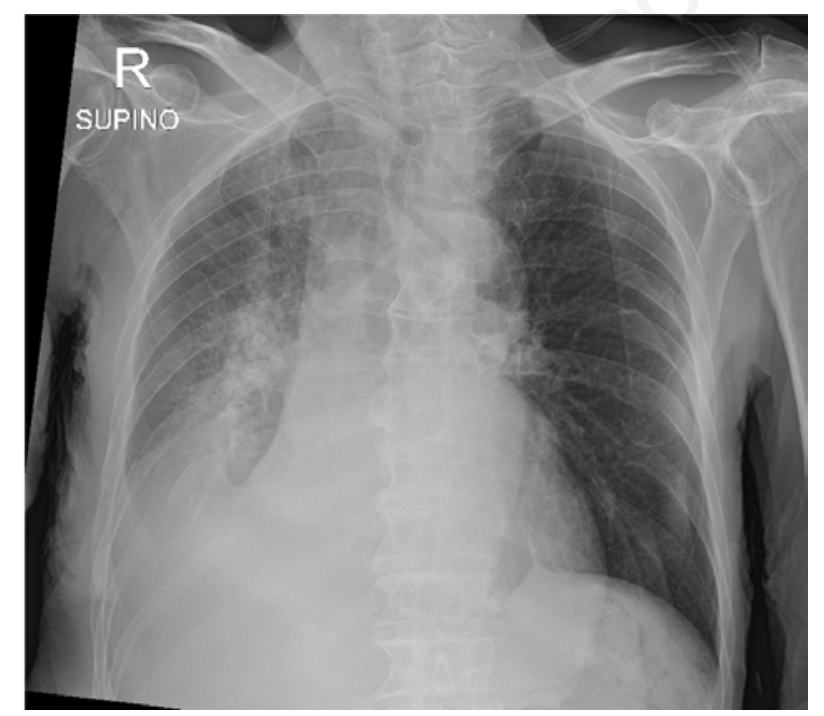

Figure 9. Chest x-ray. Right pleural effusion with mediastinal shift to the right.

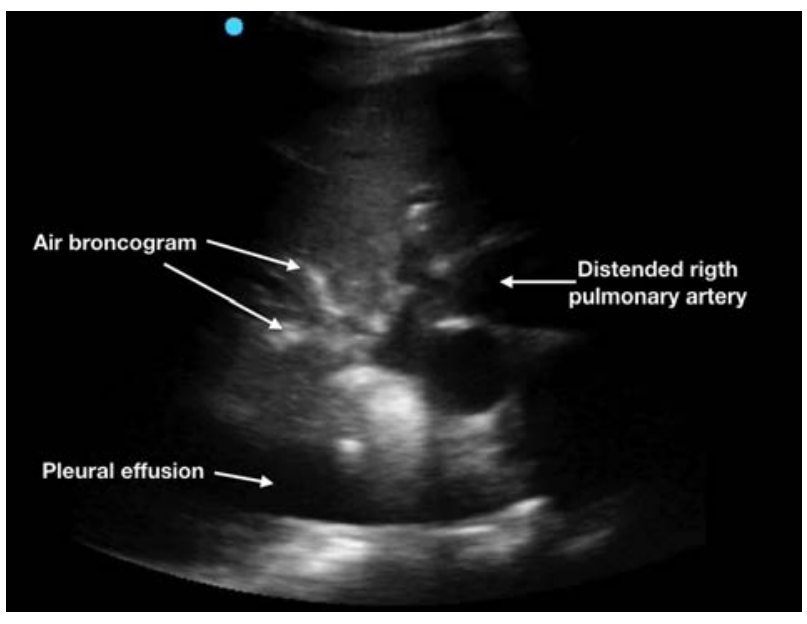

Figure 10A. US imaging. Hepatization of the right middle lung lobe with distension of the right pulmonary artery, evidence of perivascular air bronchogram and pleural effusion.

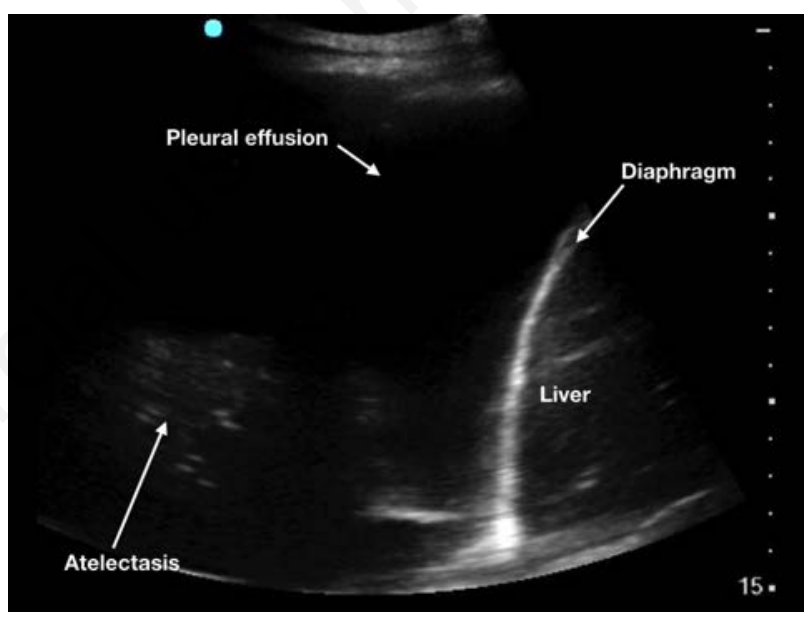

Figure 10B. US imaging. Pleural effusion with atelectasis of right inferior lung lobe.

No characteristic signs of COVID-19 pneumonia were detected, the patient was admitted to the ward and treated for bacterial pneumonia with pulmonary atelectasis ab ingestis. 


\section{Case 5}

An 85-year-old woman, CPR 7,6 mg/dL, LDH $882 \mathrm{U} / \mathrm{L}$, Ferritin 197 ng/mL (Figure 11; Figure 12A; Figure 12B; Figure 12C).

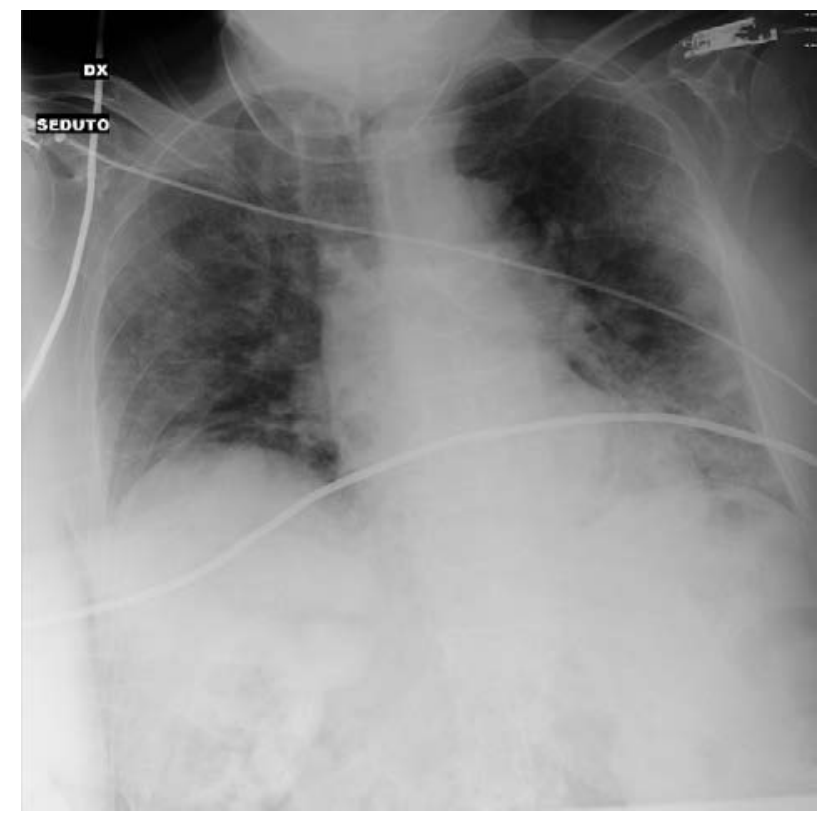

Figure 11. Chest $x$-ray. Bilateral diffuse consolidation areas extended from inferior to the upper fields with diffuse interstitial texture thickening.

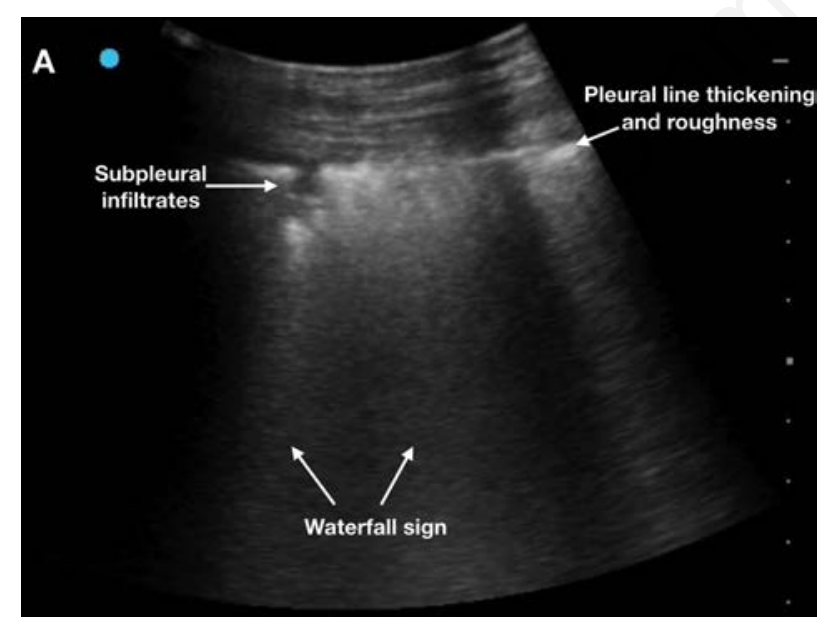

Figure 12A. US imaging. Bilateral postero-inferior thickening and roughness of the pleural line with bundled $B$ lines (waterfall sign) and subpleural infiltrates.

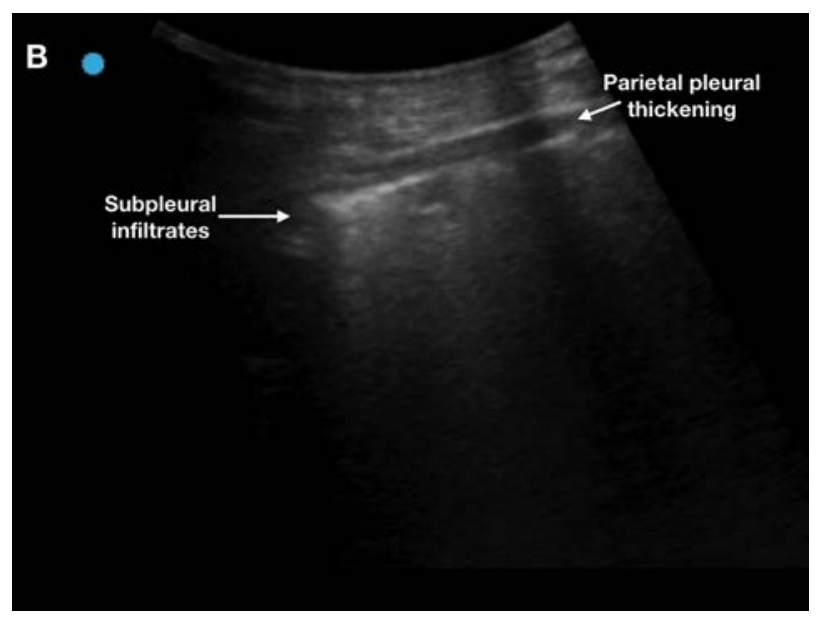

Figure 12B. US imaging. Bilateral postero-lateral hypoechoic thickening of the parietal pleura, subpleural infiltrates and thickening of the pleural line.

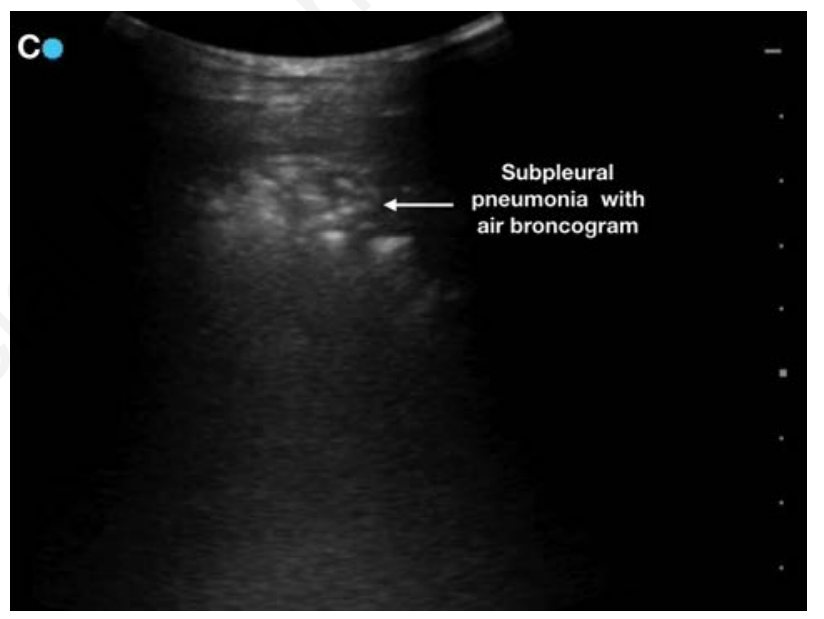

Figure 12C. Subpleural consolidation area with the presence of air bronchogram at the right postero-inferior pulmonary field. The pleural line is not detected.

On the base of chest $\mathrm{x}$-ray and US imaging, despite the second rRT-PCR results for COVID-19 was negative, the patient was transferred to a protected area. 
Case 6

An 86-year-old man, CPR 17,85 mg/dL, LDH $408 \mathrm{U} / \mathrm{L}$, Ferritin 765 ng/mL (Figure 13; Figure 14A; Figure 14B).

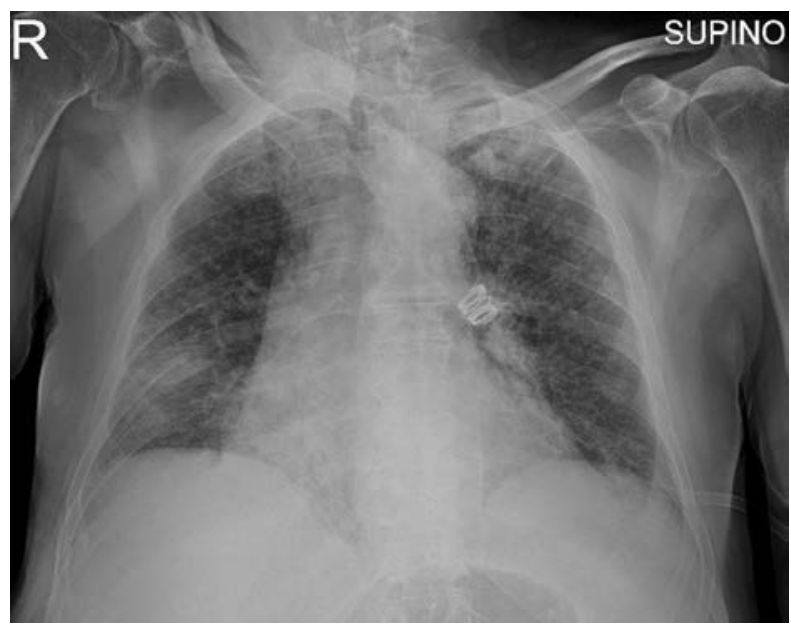

Figure 13. Chest $x$-ray. Nuanced consolidations to the inferior bilateral fields more evident on the right with interstitial texture thickening.

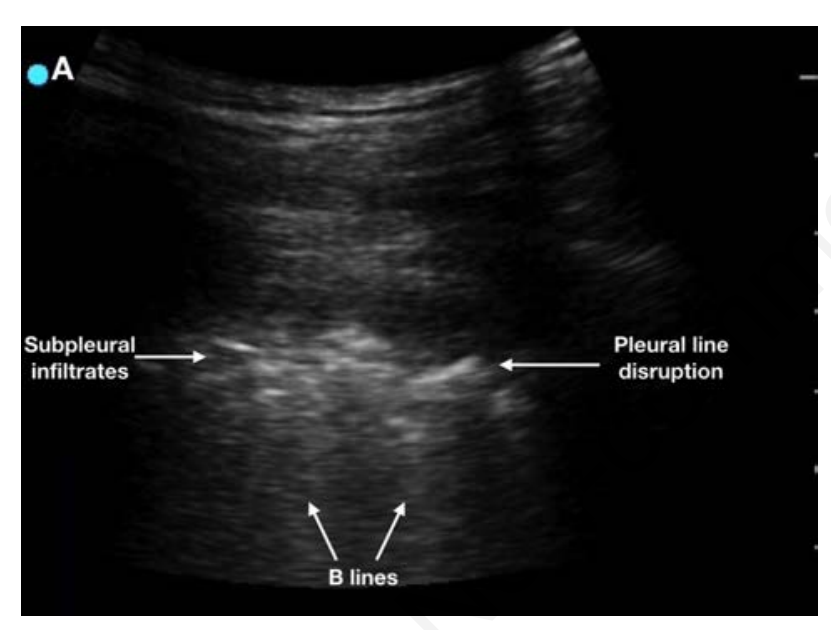

Figure 14A. US imaging. Bilateral posterior hypoechoic thickening of the parietal pleura. Subpleural infiltrates with disruption of the pleural line. Bundled B lines.

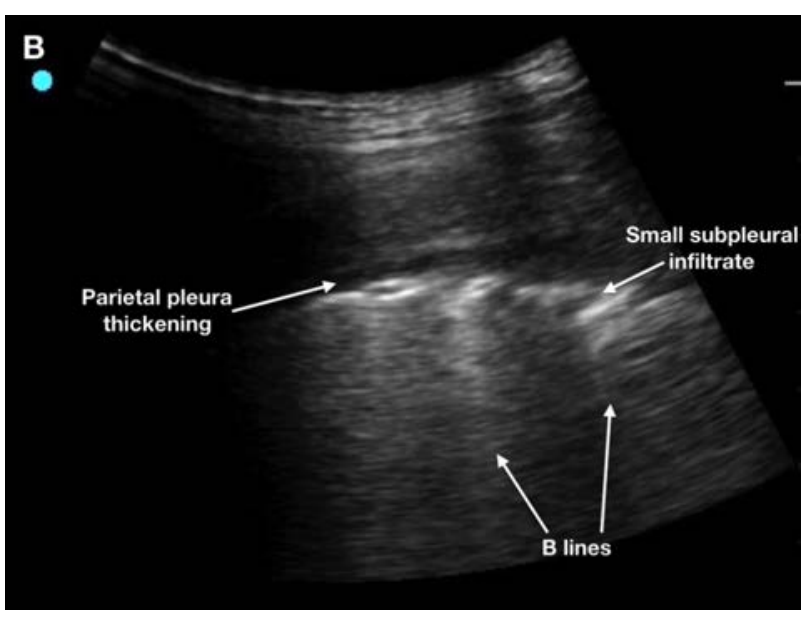

Figure 14B. US imaging. Bilateral posterior hypoechoic thickening of the parietal pleura. Subpleural infiltrates with disruption of the pleural line. Bundled B lines.

On the base of chest x-ray and US imaging, despite the second rRT-PCR results for COVID-19 was negative, the patient was transferred to a protected area.

\section{Case 7}

A 79 years old Woman, CPR 21,8 mg/dL, LDH $613 \mathrm{U} / \mathrm{L}$, Ferritin $89 \mathrm{ng} / \mathrm{mL}$ (Figure 15; Figure 16A, Figure 16B; Figure 16C; Figure16D; Figure 17).

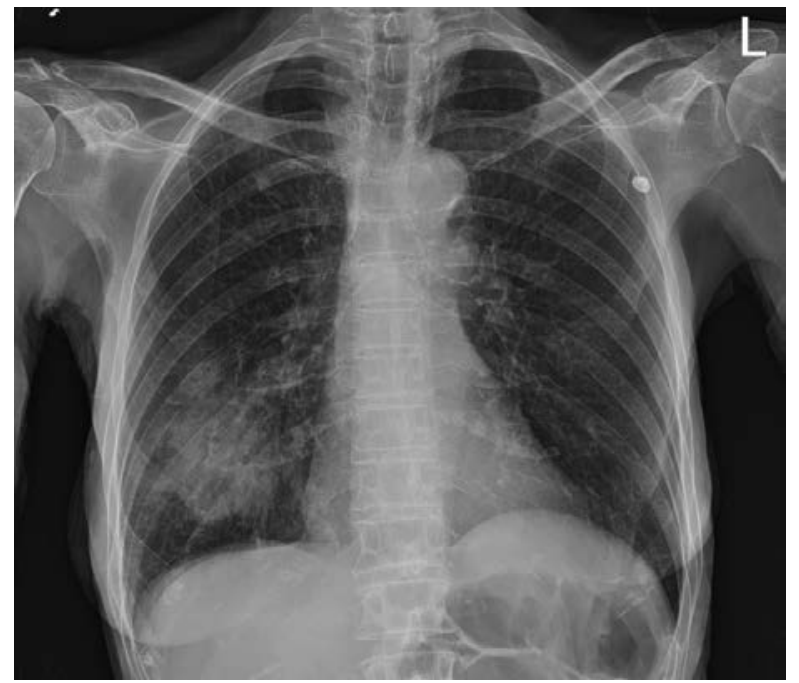

Figure 15. Chest $x$-ray. Roundish consolidation area in the right inferior field. 


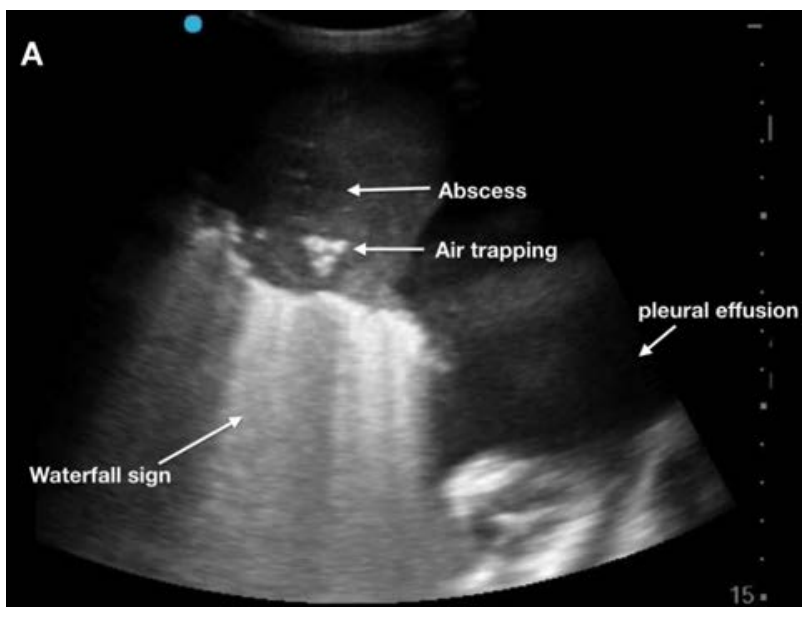

Figure 16A. US imaging. Basal anterolateral scan upwards. Hepatization of the right lower lobe with the lobar delimitation, internal hypo-isoechoic areas, and peripheral pleural effusion. Wet lung to the right middle lobe with bundled B lines starting from the fissure (waterfall sign).

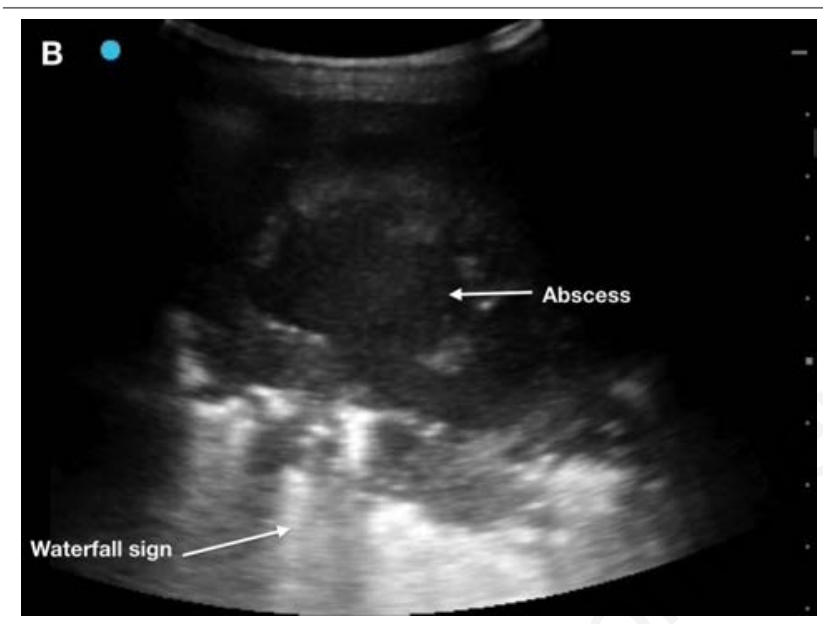

Figure 16B. Lower right lobe hepatized with a large hypo-isoechoic roundish area containing dense and corpuscular fluid material with fine movements, synchronous with the heartbeats, which, at color doppler, produce the "flash artefact" effect.

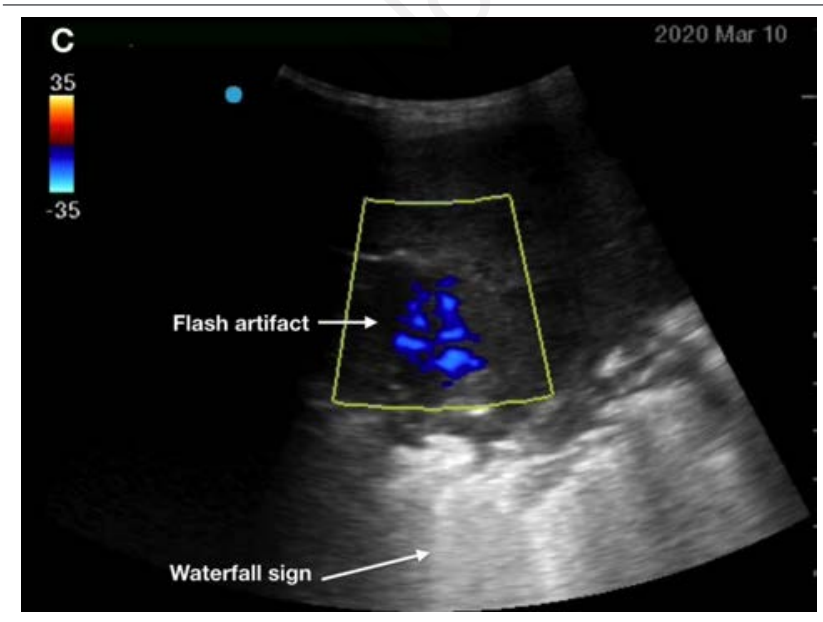

Figure 16C. Lower right lobe hepatized with a large hypo-isoechoic roundish area containing dense and corpuscular fluid material with fine movements, synchronous with the heartbeats, which, at color doppler, produce the "flash artefact" effect.

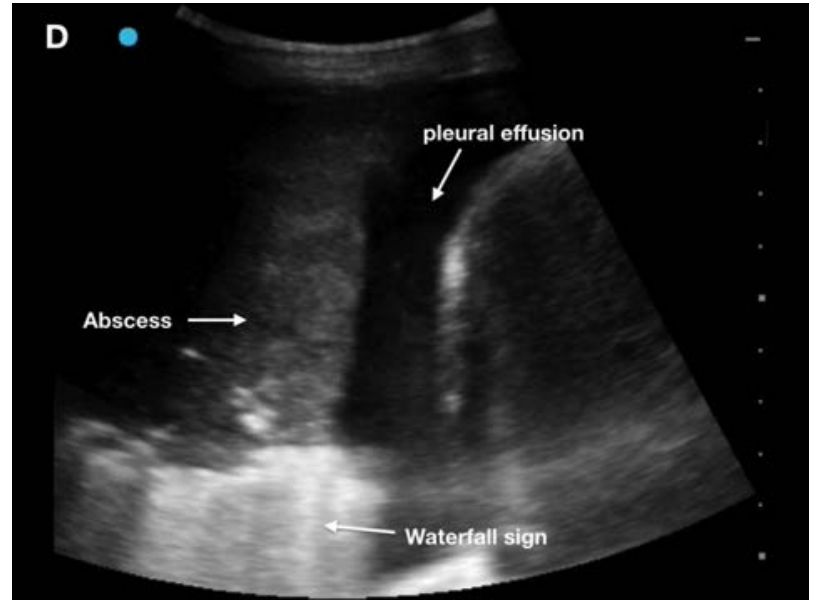

Figure 16D. Lung abscess in the pneumonia of the inferior right lobe with pleural effusion. Normal findings al left lung, no characteristic signs of COVID-19 pneumonia, the patient was admitted to the ward.

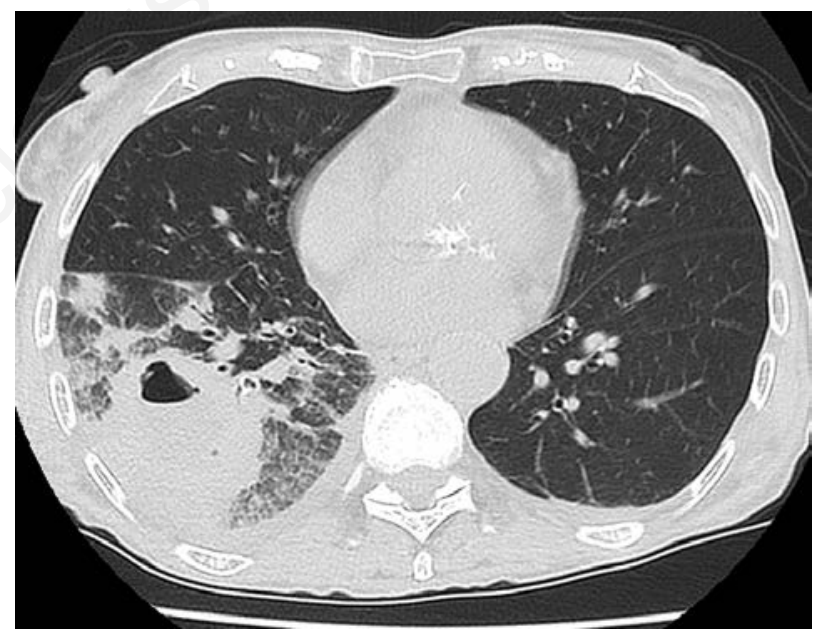

Figure 17. TC imaging. Roundish consolidation area with central cavitation to the lower right lobe of $7 \mathrm{~cm}$ in diameter with some ground glass areas around it. Pneumonia with right lung abscess at the inferior lobe.

US-guided drainage was performed with pus sampling. The culture examination of pus resulted in Staphylococcus Aureus.

Blood cultures were positive for Staphylococcus Aureus, and target antibiotic therapy was administrated. 


\section{Case 8}

A 65 -years-old man, CPR 14,7 mg/dL, LDH 637 U/L, Ferritin 295 ng/mL (Figure 18; Figure 19A; Figure 19B; 19C).

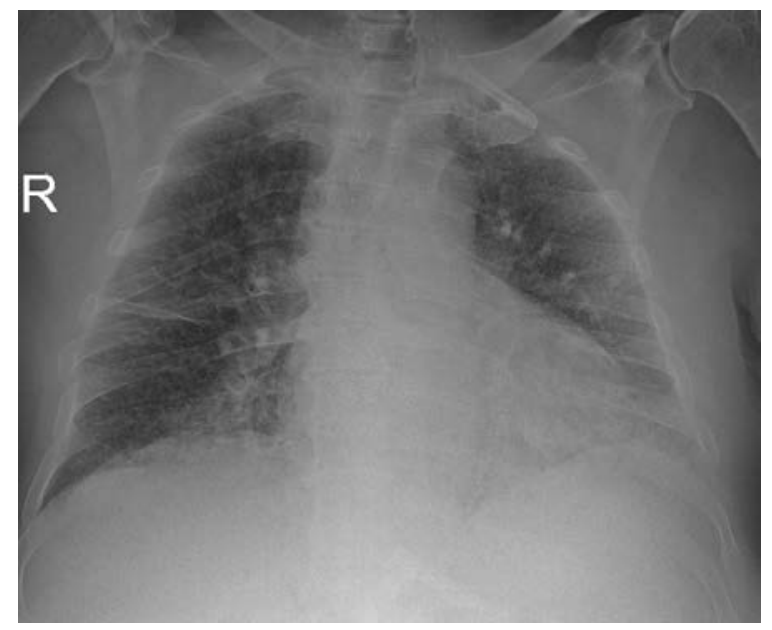

Figure 18. Chest $x$-ray. Diffuse, nuanced interstitial texture thickening, particularly evident in subpleural areas.

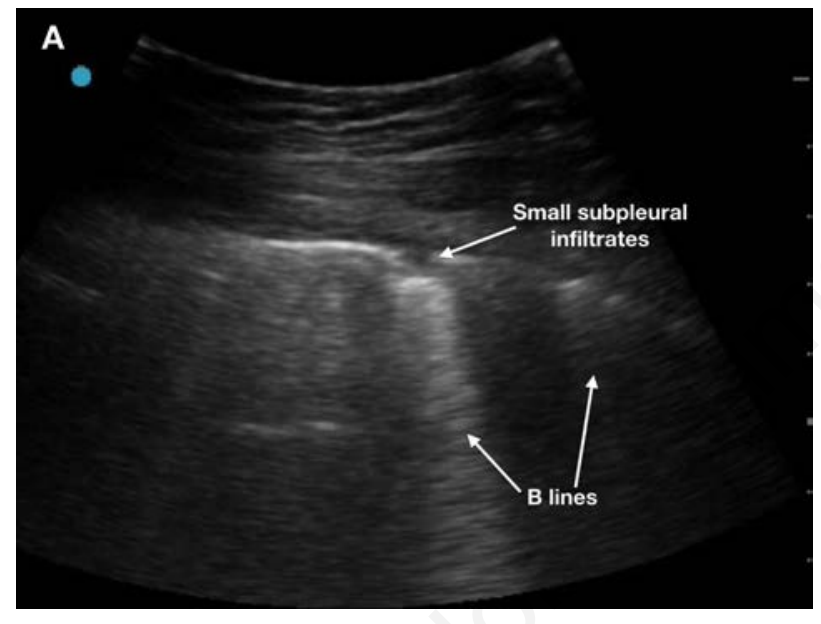

Figure 19A. US imaging. Small subpleural infiltrates that determine pleural line irregularity and interruption. The pleural line often appears thickened. B-lines departing from subpleural infiltrates.

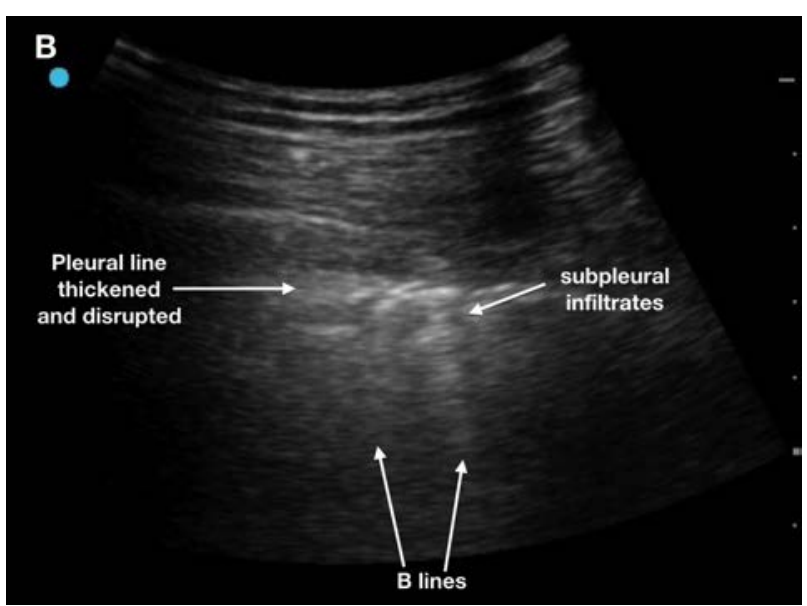

Figure 19B. US imaging. Small subpleural infiltrates that determine pleural line irregularity and interruption. The pleural line often appears thickened. B-lines departing from subpleural infiltrates.

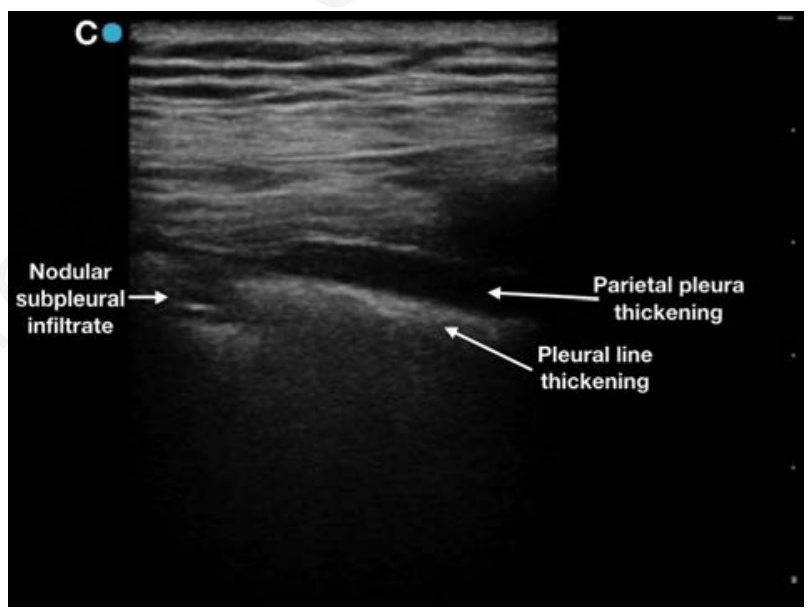

Figure 19C. Hypoechoic thickening of the parietal pleural line. Small subpleural infiltrate $(3 \mathrm{~mm})$ with air bronchogram.

Because the first rRT-PCR result for COVID-19 was negative, the patient was transferred to a protected area. Here, a second rRTPCR result was positive. 


\section{Case 9}

A 61-year-old man, CPR 3,9 mg/dL, LDH 699 U/L. History of chronic ischemic cardiomyopathy with heart failure, chronic kidney disease, and water retention (Figure 20; Figure 21A; Figure 21B; Figure 21C).

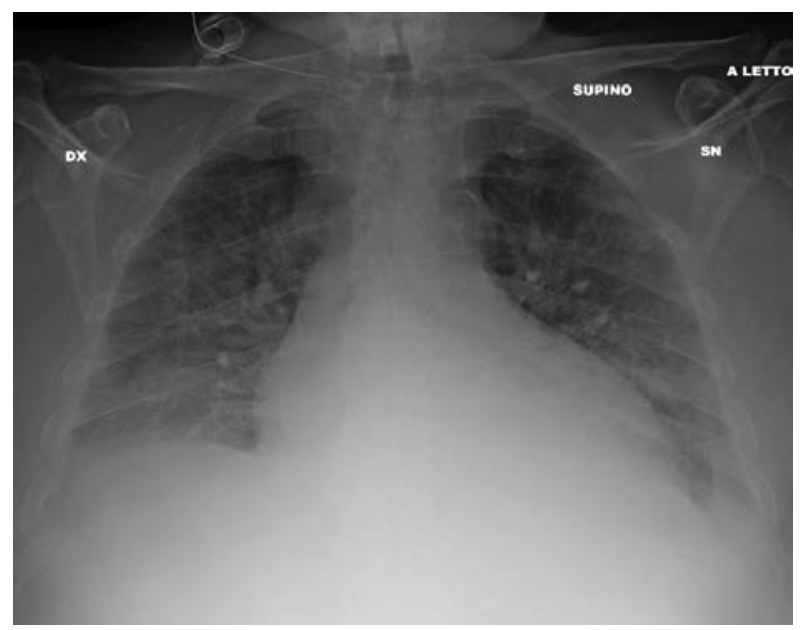

Figure 20. Bilateral hilar-para hilar interstitial-alveolar infiltrate with pleural opacities.

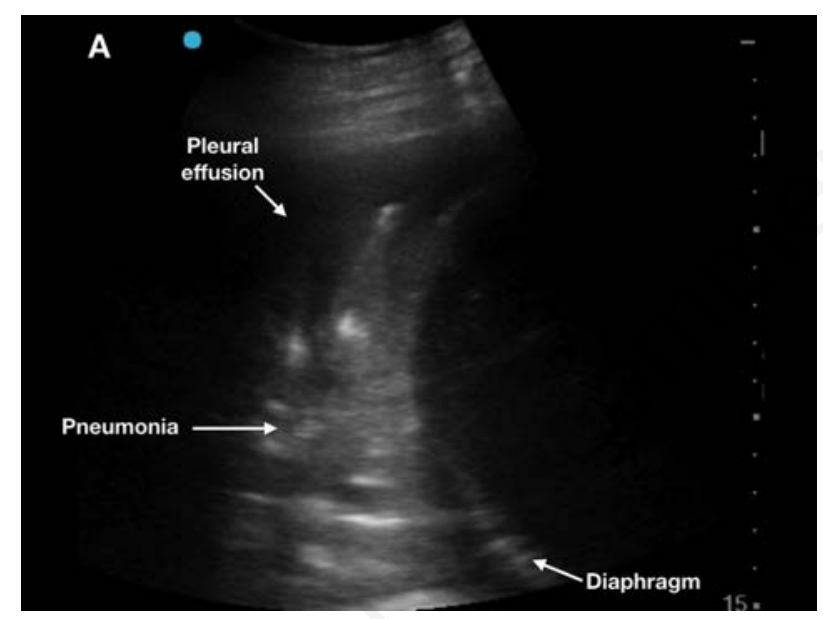

Figure 21A. US imaging. Bilateral pleural effusion prevalent on the right. After drainage of the right hemithorax appears extensive pneumonia in the lower lobe with a static air bronchogram.

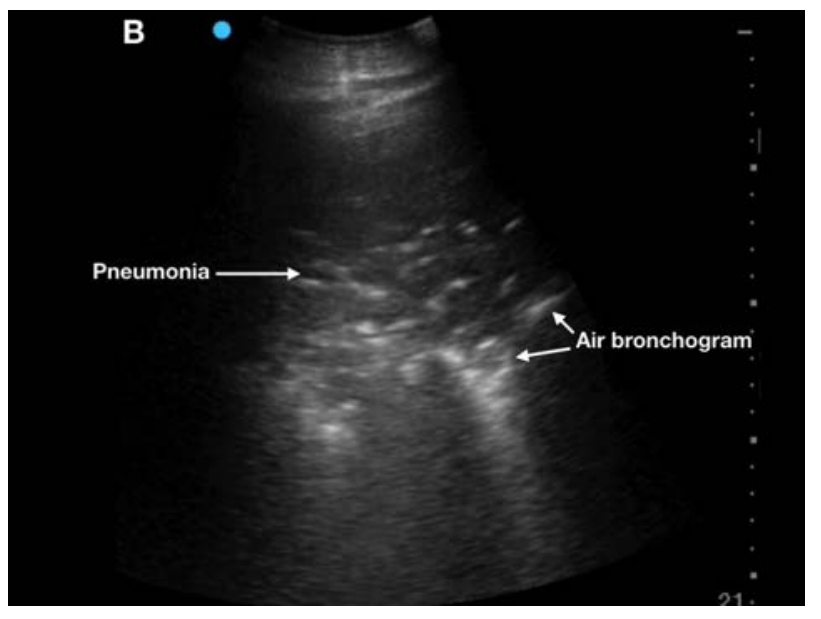

Figure 21B. US imaging. Bilateral pleural effusion prevalent on the right. After drainage of the right hemithorax appears extensive pneumonia in the lower lobe with a static air bronchogram.

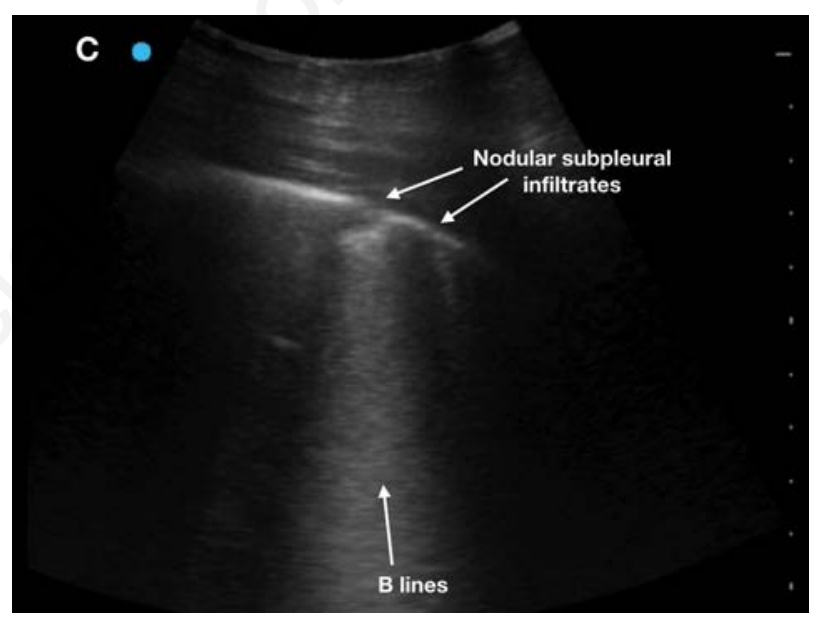

Figure 21C. On the left side, small subpleural nodules from which $B$ lines are generated, thickened pleural line with a rough surface.

On the base of the negative rRT-PCR result, the patient was maintained in the ward but a single protected room. A second rRTPCR result on swab was negative, but another rRT-PCR result on pleural effusion sample, collected by echo guided chest drain, was positive. The patient underwent respiratory and kidney failure and died after a few days. 


\section{Case 10}

A 75 years old woman, WBC 10700/mL, CPR 19,8 mg/dL, LDH 564 U/L, Ferritin 121 ng/mL (Figure 22; Figure 23A; Figure 23B; Figure 24A; Figure 24B; Figure 24C).

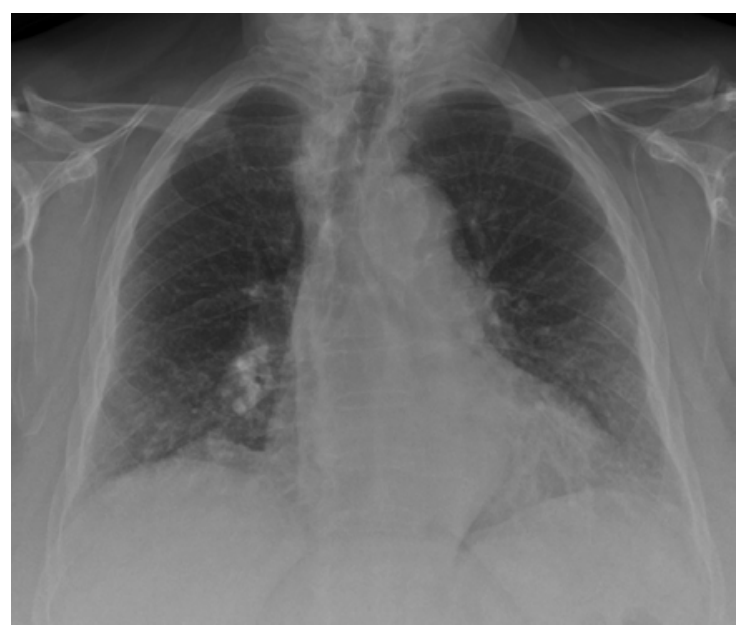

Figure 22. Chest TC. Thickening of the broncho-vascular texture more evident to the lung bases.

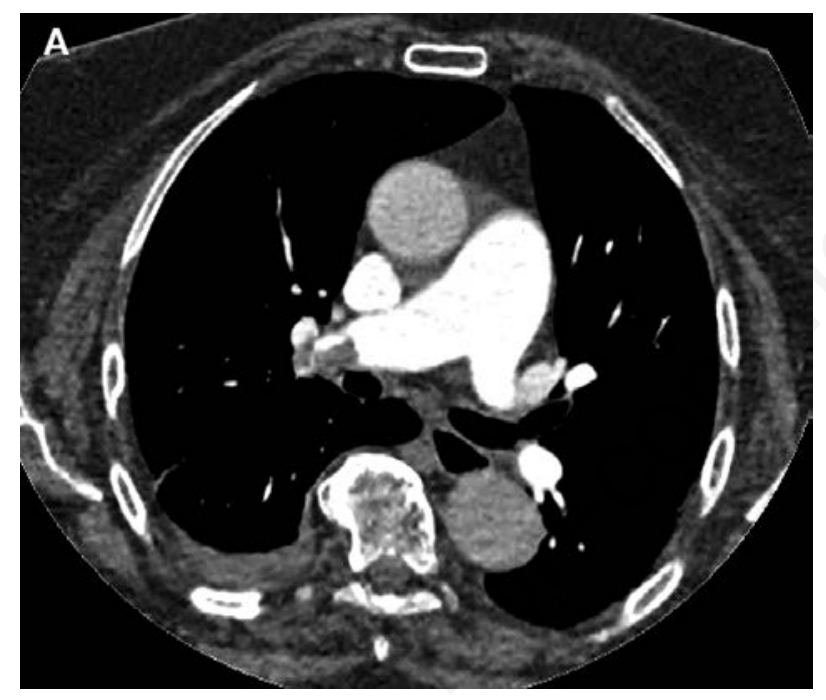

Figure 23A Endoluminal defects at the lobar, segmental and subsegmental branches of the right pulmonary artery for thromboembolism. Concomitant small pleural effusion close to infiltrates.

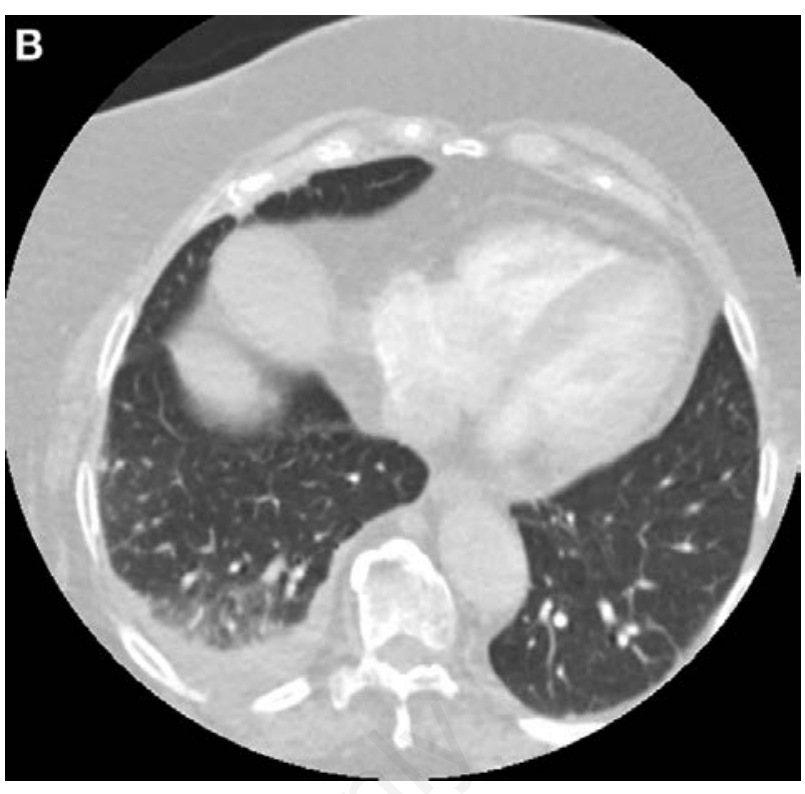

Figure 23B. Endoluminal defects at the lobar, segmental and subsegmental branches of the right pulmonary artery for thromboembolism. Concomitant small pleural effusion close to infiltrates.

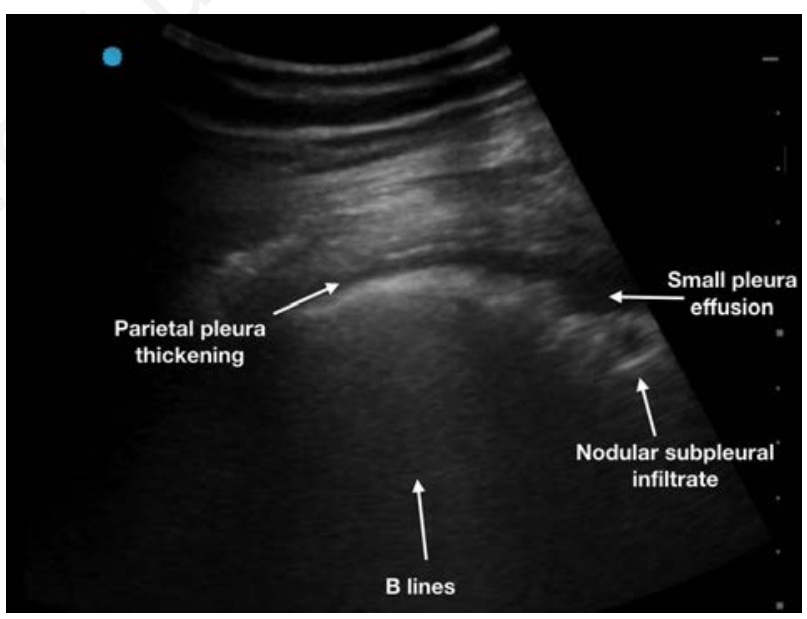

Figure 24A. US imaging. Hypoechoic thickening of the parietal pleura with adjacent small pleural effusion film. Thickened pleural line with the irregular and interrupted surface due to the presence of small hypoechoic subpleural nodular infiltrates. 


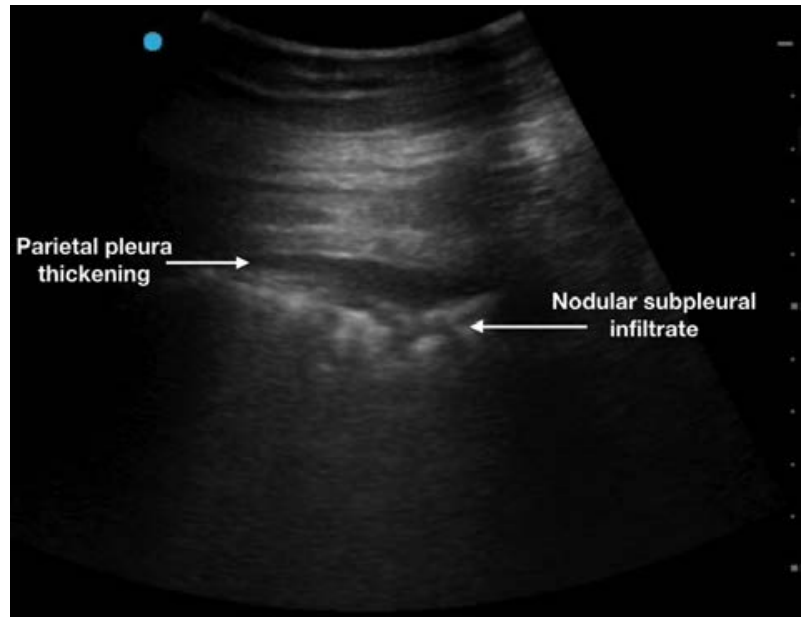

Figure 24B. US imaging. Hypoechoic thickening of the parietal pleura with adjacent small pleural effusion film. Thickened pleural line with the irregular and interrupted surface due to the presence of small hypoechoic subpleural nodular infiltrates.

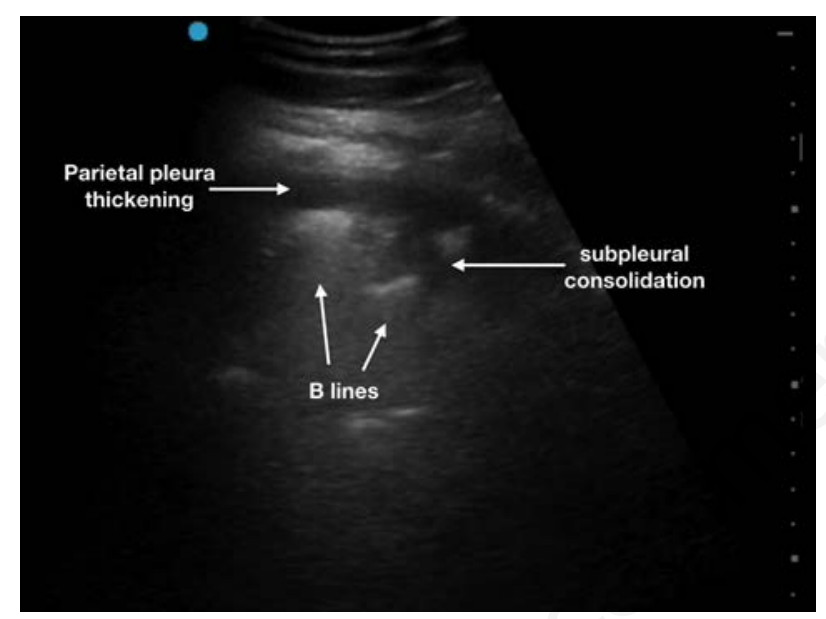

Figure 24C. B lines and inhomogeneous hypoechoic subpleural consolidation with small echogenic spots (air bronchogram).

Based on the CT and ultrasound findings, despite the negative swab, the patient was transferred to a protected area where a second rRT-PCR result on swab was positive.

\section{Case 11}

An 81 years old man, WBC 24200/mL, CPR 9,2 mg/dL, Procalcitonin $1,17 \mathrm{ng} / \mathrm{mL}$

The patient was transferred to the ward from a protected area where he had been admitted for suspected COVID-19 pneumonia, 2 swabs had been carried out with negative results; moreover, the procalcitonin dosage was high, directing the diagnosis towards a form of bacterial pneumonia (Figure 25).

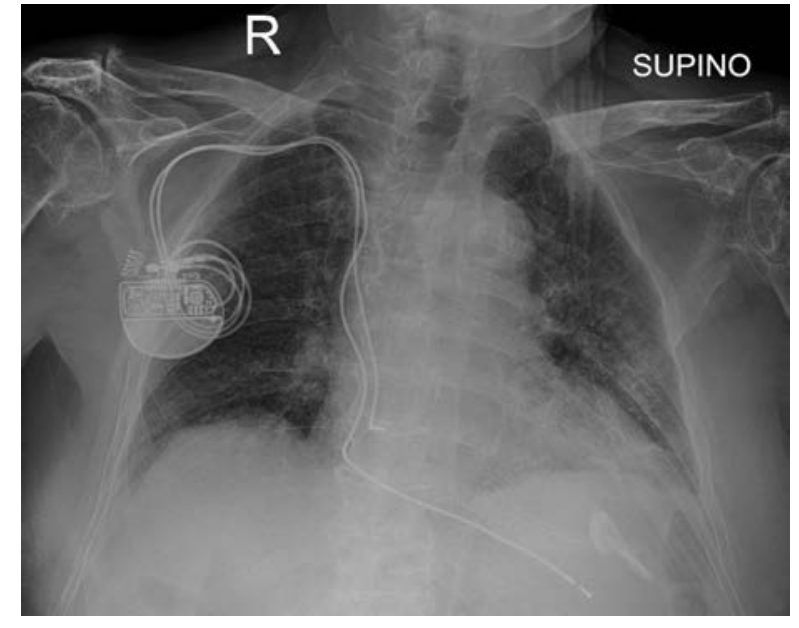

Figure 25. Chest $x$-ray. Diffuse, interstitial texture thickening. Small middle-basal consolidation on the left. Cardiac PM on the right side.

Despite the antibiotic treatment, the patient did not improve (Figure 26A; Figure 26B).

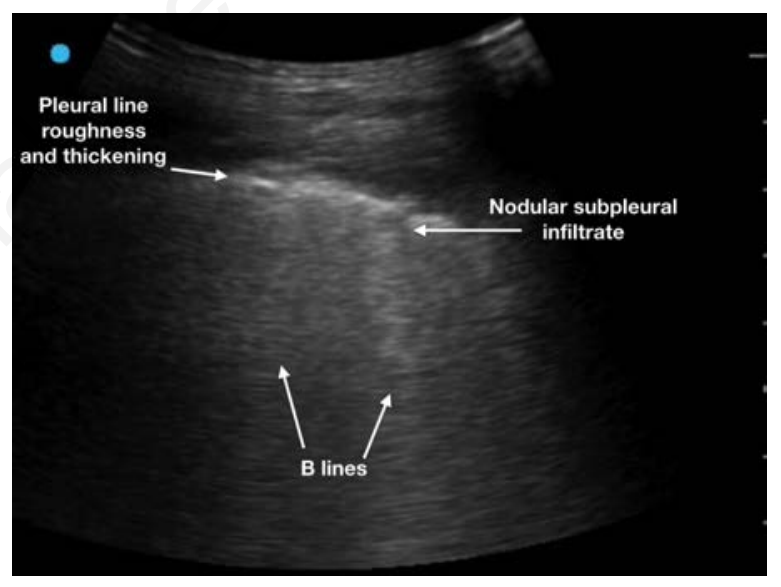

Figure 26A. US imaging. On the right inferior posterior-lateral side, thickening and coarseness of the pleural line interrupted by small subpleural infiltrates with numerous $B$ lines.

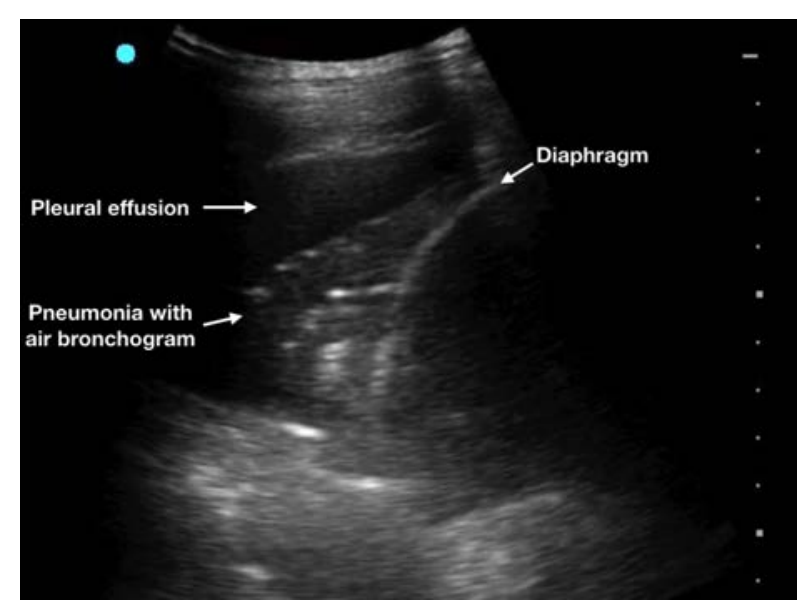

Figure 26B. On the left posterolateral supradiaphragmatic area, there is pneumonia with a gross consolidation area with an air bronchogram and parapneumonic pleural effusion. 
A sample of pleural effusion was collected under ultrasound guidance and sent for COVID-19 rRT-PCR, which was positive. The patient was transferred back to a protected area.

\section{Discussion}

The average diameter of SARS-CoV-2 is about $120 \mathrm{~nm}$, so it is speculated that the virus particles can be inhaled into the airway and lungs, even into the alveoli. This fact may explain why the lesions of viral pneumonia are mainly in the subpleural areas. What has been clear is that COVID-19 generally begins in the terminal alveoli, which are close to the pleura and can be clearly observed by LUS. ${ }^{10}$

So, it could be possible that in some phases of the disease, the patient has a negative nasopharyngeal swab and even a negative bronchoalveolar lavage (BAL). However, he still has an acute manifestation of the lower respiratory tract, as we found in case 3 .

Pleural effusion is not a characteristic ultrasound lesion of COVID-19 pneumonia, often only a thin film of liquid is observed close to pleural thickenings and subpleural consolidations. . $^{2,3}, 10$ When it is detected in patients with other diseases (such as heart failure, inflammatory or neoplastic diseases, etc.), this quickly becomes infected when COVID-19 pneumonia is present, as we found in case 9 .

Sometimes pleural effusion is detectable in the advanced stage of the disease in conjunction with massive lung consolidations as we found in case 11 .

These findings clearly show how LUS plays an important role together with the chest $\mathrm{x}$-ray in identifying patients with interstitial pneumonia from COVID-19. This approach could help to place patients correctly in protected hospital areas avoiding further spreading of the infection within the hospital wards and reducing exposure to the virus by healthcare professionals. In fact, 9/11 patients had typical LUS findings for COVID-19, but only $3 / 11$ patients had a second positive nasopharyngeal swab, and 2/11 had positive swab on pleural fluid. 6/11 patients remained negative with strongly suspicious LUS lesions, and so treated and isolated as Covid-19 positive. 2/11 had negative swab and none LUS findings, thus treated as affected by other pathologies.

Finally, the control of white blood cells and procalcitonin could further help in distinguishing lung diseases at the admission of the patient. ${ }^{1}$ However, these findings cannot exclude the concomitant presence of the COVID-19 infection as we have described in our last case.

\section{Conclusions}

LUS performed at bedside during outbreak, allowed the easy detection of characteristic features of COVID-19 pulmonary infec- tion. It assumes a great importance in order to select the right path to propose for the patient admitted to ED who, despite having negative results for the nasopharyngeal swab, should instead be directed to protected areas reserved for COVID-19 infected patients avoiding further spreading of the infection within the hospital wards and reducing exposure to the virus by healthcare professionals. Further studies are needed for evaluating if long-term complications (such as pleural effusions, empyema, lymphadenopathy, and lung cavitation) will arise or whether the detection of these features, as in our cases, is attributable to lung diseases other than COVID19 infection.

\section{References}

1. Huang C, Wang Y, Li X, et al. Clinical features of patients infected with 2019 novel coronavirus in Wuhan, China. Lancet 2020;395:497-506.

2. $\mathrm{Zu} \mathrm{Z}$, Jiang MD, Xu PP, et al. Coronavirus Disease 2019 (COVID-19): A Perspective from China. Radiol 2020; doi: https://doi.org/10.1148/radiol.2020200490.

3. FanY, Zhang H, Xie J, et al. Sensitivity of Chest TC for COVID-19: Comparision to RT-PCR. Radiology 2020: 200432. doi: 10.1148/radiol.2020200432.

4. Pan F, Ye T, Sun P, et al. Time course of lung changes on chest CT during recovery from 2019 novel coronavirus (COVID-19) pneumonia. Radiology 2020:200370. doi: 10.1148/radiol. 2020200370.

5. Mojoli F, Bouhemad B, Mongodi S, Lichtenstein D. Lung ultrasound for critically ill patients. Am J Respir Crit Care Med 2019;199:701-14. doi: https://doi.org/10.1164/rccm.2018020236CI.

6. Mayo PH, Copetti R, Feller-Kopman D, et al. Thoracic ultrasonography: a narrative review. Intensive Care Med 2019;45(9):1200-11.

7. Soldati G, Demi M, Smargiassi A, et al. The role of ultrasound lung artifacts in the diagnosis of respiratory diseases. Expert Rev Respir Med 2019;13:163-72. doi: 10.1080/ 17476348.2019 .1565997$.

8. Yang Y, Wang Z. Application of pulmonary ultrasound in severe H7N9. Chinese J Med Ultrasound 2019;: 72-6.

9. Soldati G, Smargiassi A, Inchingolo R, et al Proposal for international standardization of the use of lung ultrasound for patients with COVID-19. A simple, quantitative, reproducible method. J Ultrasound Med 2020. doi: https://doi.org/10.1002 /jum.15285.

10. Huang Y, Wang S, Liu Y, al. A preliminary study on the ultrasonic manifestations of peripulmonary lesions of non-critical novel coronavirus pneumonia (COVID-19). SSRN 2020. doi: https://dx.doi.org/10.2139/ssrn.3544750 